\title{
Influence of the channel arrangement for the passage of the coolant on the heat transfer process of a convective heater
}

\author{
Samvel Sargsyan ${ }^{*}$ and Viktoria Borisova \\ Moscow State University of Civil Engineering, Yaroslavskoe shosse, 26, Moscow, 129337, Russia
}

\begin{abstract}
The availability of highly economical indicators of convective type heating appliances, as well as the wide use of window sills, solid glazing of walls in the construction equipment, and the expansion of the use of convective (ribbed) type heating appliances, taking into account the possible improvement of their sanitary and hygienic and heat engineering properties. Constructions of convective type (convector) type heating devices with rather low heat engineering indicators. To improve the thermo-technical qualities of convectors, special attention is paid to the determination of the optimum dimensions of the fin, the channel for the passage of the coolant, the fin length, the optimum shape of the rib, and so on. The task of this work is to find new ways that require the heat transfer process of ribbed heating devices. The problem of the optimal distribution of the heat source (channel for the passage of the coolant) relative to the horizontal axis of symmetry of the rib is considered. Analytical dependences are obtained, which allow to determine the local values of heat transfer changes for nonisothermal plates, the temperature change at which is a step-change function. The process of convection in a laminar boundary layer is shown as a process of nonstationary thermal conductivity of a semi-boundary body. When solving the problem of radiant heat exchange between the ribs and surrounding surfaces, and between the edge and the environment. The directions of the experimental study are presented with the purpose of determining: the thickness of the boundary layer near the surface of the rib; the nature of the distribution of temperature along the edge with different locations of the source of heat; thermal engineering studies on convectors, various measurements of the channel for the passage of the coolant, various finning steps, and various fin sizes.
\end{abstract}

\section{Theoretical and practical foreword issues}

The task of this work is to find new ways to intensify the heat transfer process of ribbed heating devices, in particular convectors. To this end, the question of the optimal location of the heat source (channel for the passage of the coolant) was considered relative to the horizontal axis of symmetry of the rib (Figure 1).

Corresponding author: SargsyanSV@mgsu.ru 
As is known, the surface of a fin operates unevenly with the central location of the heat source. Heat transfer of the upper part of the rib is much less than the bottom, where cooler air contacts the plate. As a consequence, the isotherms obtained on the surface of the rib are shifted from the center and have the form of an elongated one at the top. Such a picture of the location of the isotherms leads to the assumption that the location of the heat source with eccentricity with respect to the central horizontal axis can have either a positive or negative influence, depending on how correctly the choice of eccentricity was made.
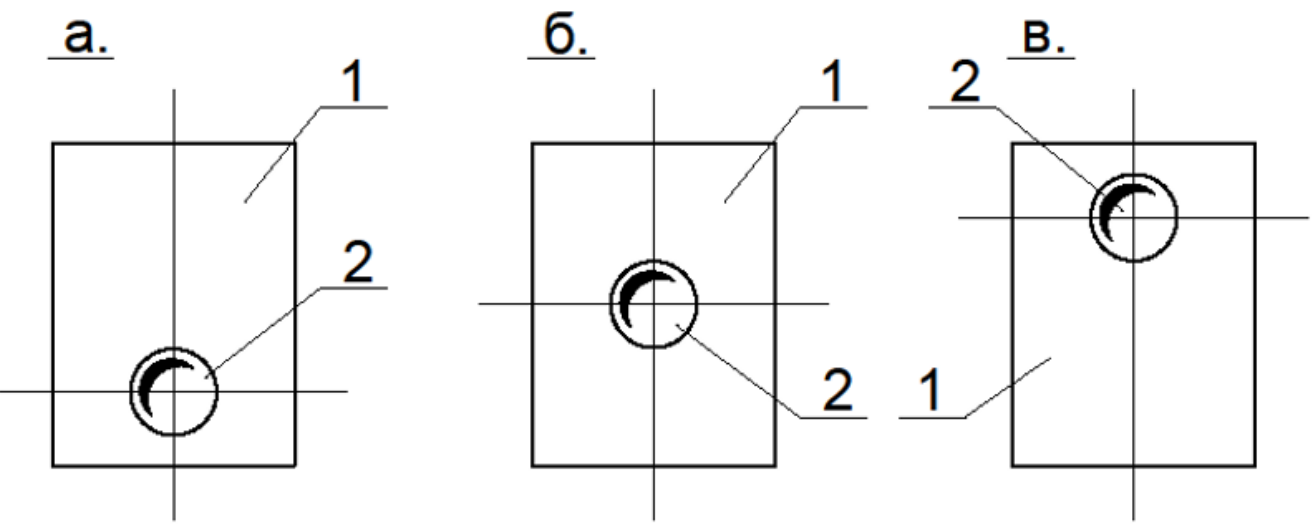

Fig. 1. Location of the heat source relative to the horizontal axis of symmetry: 1- edge; 2 - channel for the passage of the coolant (a.below the center of the plates; 6 . in the center of the plates; в. above the center of the plates)

A study on the expediency of the eccentric location of the heat source showed that existing opinions are contradictory. A number of authors find the optimal location of the source of heat with eccentricity down, while others, on the contrary, are up. There is a need to find the right solution to the task. In connection with this, the goal of this paper is to identify the optimal location of the heat source with respect to the horizontal axis of symmetry of the rib.

At the same time, it is necessary to determine the optimal location of the fin pitch. It is assumed that the optimal distance between the plates (fin pitch) is a function of the maximum thickness of the boundary layer. The value of the thickness of the boundary layer should be determined taking into account the non-isothermicity of the surface of the edge [1-4].

\section{Method and order of research behavior}

To solve the problems, the following method and order of research was chosen: investigation of the heat transfer process of the rib to obtain analytical expressions that allow to determine the heat transfer of the rib in the presence of a picture of the temperature field; finding a picture of the temperature field for finned surfaces with different locations of the source of heat; Determination of heat transfer by the nature of the change in the temperature of the surface of the rib.

\section{Analytical study}

The transfer of heat through finned surfaces is carried out by processes of internal heat perception, thermal conductivity and external heat transfer. Corresponding to these processes, the value of the heat flux $(\mathrm{W})$ can be calculated from the expressions: 


$$
\begin{aligned}
& Q=\alpha_{1} A_{1}\left(\mathrm{t}_{6,1}-\mathrm{t}_{w, 1}\right), \\
& Q=\frac{\lambda}{\delta} A_{1}\left(\mathrm{t}_{w, 1}-\mathrm{t}_{w, 2}\right), \\
& Q=\alpha_{2} A_{2}\left(\mathrm{t}_{\mathrm{w}, 2}-\mathrm{t}_{\mathrm{B}, 2}\right),
\end{aligned}
$$

where: $\alpha_{1}$ and $\alpha_{2}$ are the coefficients of heat perception and heat transfer respectively, $\mathrm{W}$ $/\left(\mathrm{m}^{2}{ }^{\circ} \mathrm{C}\right) ; \mathrm{A} 1, \mathrm{~A} 2$ - the area of the surfaces of heat perception and heat transfer, $\mathrm{m}^{2} ; \mathrm{t}_{\mathrm{B}, 1}, \mathrm{t}_{\mathrm{B}}$, 2 - temperature of the heat carrier and air surrounding the medium plate, ${ }^{\circ} \mathrm{C} ; \mathrm{t}_{\mathrm{w}, 1}, \mathrm{t}_{\mathrm{w}, 2}-$ temperature of heat-sensing and heat-dissipating surfaces, ${ }^{\circ} \mathrm{C} ; \lambda$ is the thermal conductivity of the fin material, $\mathrm{W} /\left(\mathrm{m}^{\circ} \mathrm{C}\right) ; \delta$ is the thickness of the rib, $\mathrm{m}$.

These heat flow equations are very approximate and are based on the assumption of a constant temperature and a heat transfer coefficient $\alpha$ over the edge surface. In fact, both the temperature and the value of the heat transfer coefficient over the edge vary over large limits.

The process of external heat transfer is a process of free convection along a nonisothermal vertical plate. As the initial data there is a character of the distribution of isotherms on the plate, obtained experimentally. The method for calculating the amount of heat flux by the process of external heat transfer allows one to take into account the variability in the value of the heat transfer coefficient $\alpha$ and the character of the temperature distribution over the surface of the rib, while the calculation of the heat flux in the heat conduction process does not take into account the features of the external heat transfer process, in particular the variable character of the heat transfer coefficient $\alpha$.

The process of external heat transfer is characterized by the fact that free convection takes place along the nonisothermal plate. And the temperature along any section of the edge changes along a curve that does not obey the general pattern [5-10].

The convection process in a laminar boundary layer can be represented as a process of nonstationary heat conductivity of a semibounded body whose differential equation has the following form:

$$
\frac{\partial t}{\partial \tau}=a \frac{\partial^{2} t}{\partial y^{2}}
$$

where: $t$ is the air temperature in the boundary layer at a distance $y$ from the surface of the plate and at a distance $x$ from the lower edge.

For an isothermal plate with boundary conditions

$$
\left\{\begin{array}{llll}
t=t_{\text {s }}=\text { const } & \text { npu } & \tau=0, & y \succ 0 ; \\
t=t_{w}=\text { const } & n p u & \tau \succ 0, & y=0 ;
\end{array}\right.
$$

for a nonisothermal plate with boundary conditions

$$
\left\{\begin{array}{llll}
t=t_{\text {s }}=\text { const } & \text { npu } & \tau=0, & y \geq 0 ; \\
t=t_{w}=f(\tau) & \text { npu } & \tau \succ 0, & y=0 ;
\end{array}\right.
$$

Here the parameter $\tau$ (time) replaces the ratio $x / \varepsilon_{1} u_{s}$ for uniform forced motion or $2 x^{1 / 2} / \varepsilon_{2} \sqrt{2 g \beta \theta_{w}}$ for free convection. In these expressions, $\varepsilon 1$ and $\varepsilon 2$ take into account the assumed assumption about the replacement of a viscous fluid by a discrepancy. An analysis of the convection process in the laminar boundary layer and the process of nonstationary thermal conductivity showed that such a substitution is possible when $\varepsilon_{1}=0,346 \operatorname{Pr}^{-\frac{1}{3}}$ and $\varepsilon_{2}=1,15(0,952 \operatorname{Pr})^{-\frac{1}{2}}$. In the presented dependences of the $\operatorname{Pr}$ Prandtl test, and us, the velocity of air movement outside the boundary layer. 
The convection process in the laminar boundary layer can be represented by the equation (4) if the parameter $\tau$ obeys the following regularities:

for forced convection

$$
\tau=2,886 \operatorname{Pr}^{-\frac{1}{3}} \frac{x}{u_{s}}
$$

with natural convection

$$
\tau=1,232(0,952+\operatorname{Pr})^{-\frac{1}{2}}\left(\mathrm{~g} \beta \theta_{w}\right)^{-\frac{1}{2}} x^{\frac{1}{2}} .
$$

Integrating equation (4) with the boundary conditions (6), we can solve the problem for a nonisothermal plate. For the case of a stepwise change in temperature, the solution takes the following form:

$$
t=t_{\theta} \operatorname{erf}\left(\frac{y}{2 \sqrt{a \tau}}\right)+t_{1} \operatorname{erfc}\left(\frac{y}{2 \sqrt{a \tau}}\right)+\ldots . .+\left(t_{m}-t_{m-1}\right) \operatorname{erfc}\left(\frac{y}{\left.2 \sqrt{a\left(\tau_{m}-\tau_{m-1}\right.}\right)}\right) .
$$

Expression (9) is the law of variation of air temperature in the laminar boundary layer at time $\tau$, corresponding to the distance $x$. Depending on the type of motion, this correspondence is found from expression (7) or (8).

Substituting the value of the air temperature outside the boundary layer and solving equation (9) with respect to $y$, we can determine the thickness of the thermal boundary layer.

The value of the heat flux is defined as

$$
q_{n}=-\lambda\left(\frac{\partial t}{\partial y}\right)_{y=0}
$$

After transformation of equation (10), one can obtain

$$
q_{n}=-\frac{\lambda}{\sqrt{\pi a}} \sum_{m=1}^{n} \frac{t_{m}-t_{m-1}}{\sqrt{\tau_{n}-\tau_{m-1}}}
$$

Equation (11) allows us to determine the local value of the heat flux at the point $\mathrm{x}_{\mathrm{n}}$ or at the time $\tau_{\mathrm{n}}$, which for forced convection is determined by expression (7) and for natural convection by expression (8).

The amount of heat transmitted by radiant heat exchange by the surface of the rib to the surfaces of the environment is defined as

$$
Q=\varepsilon_{n} C_{0} A_{p}\left[\left(\frac{T_{p}}{100}\right)^{4}-\left(\frac{T_{c p}}{100}\right)^{4}\right] \varphi_{p . c .}
$$

where: $\varepsilon_{\Pi}$ - reduced degree of blackness of the system; $\mathrm{C}_{0}$ is the emissivity of an absolutely black body; $A_{p}$ is the calculated radiating surface; $T_{p}$ is the temperature of the surface of the $\mathrm{rib} ; \mathrm{T}_{\mathrm{cp}}$ is the ambient temperature; $\varphi_{\text {p.c. }}$ is the irradiance coefficient between the rib and the surrounding surfaces; $\varphi_{\text {p.p. }}=1-\varphi_{\text {p.c. }}$ is the irradiance coefficient between two parallel edges.

\section{Laboratory research}

Laboratory studies are conducted with the aim of: determining the thickness of the boundary layer; identification of the distribution of isotherms on the surface of the rib, with different locations of heat sources; heat engineering studies of convective type heating appliances with different channel arrangements for coolant passage [11, 12]. 


\section{Conclusions}

In calculations for the design of convective type radiators, it is necessary to take into account that the coefficient of heat transfer from the external surface of the rib to the external medium varies along the surface gradient.

Heat transfer from the surface of the rib into the environment is determined not only by the average temperature of its surface, but also by the nature of the temperature distribution.

Calculation of the value of the heat flux taking into account the nature of the temperature distribution and the inconstancy of the value of the heat transfer coefficient of the edge is possible only in the case when the calculation is carried out by the process of external heat transfer.

A formula is given for calculating the surface density of the heat flux, in the form of free convection along a nonisothermal plate, from the surface of the rib to the surrounding medium.

An expression is given for determining the thickness of the boundary layer, taking into account the nature of the distribution of the temperature field in the laminar boundary layer, for nonisothermal plates.

The minimum pitch of fins should be determined as a function of the maximum thickness of the boundary layer.

To increase the surface density of the heat flow in convective type radiators, the source of heat (the channel for the passage of the coolant) should be located above the center of the plates.

\section{References}

1. GOST 31311-2005. Heating devices. General specifications. Moscow/2005.

2. GOST R 53583-2009. Heating devices. Test methods. Moscow/2009.

3. STO NP "AVOK" 4.2.2.2006. Radiators and convectors heating. General specifications. - M.: ABOK-PRESS, 2006.

4. Bogoslovskiy V.N. Building thermophysics (thermophysical basis of heating, ventilation and air conditioning): Moscow: 2013. - 416 pages

5. Isachenko VP, Osipova VA, Sukomel AS Heat transfer. - M.: Publishing house "Energy", 1981.

6. V.A. Zhila, S.V.Sargsyan. Engineering systems of buildings and structures. Heat and ventilation. Moscow: 2014. - 320 pages

7. V.I. Sasin On the problem of reducing the design parameters of the coolant in heating systems // Akvaterm. - 2002. - No. 1. - P. 24-26.

8. V.I. Sasin, G.A. Bershidsky, T.N. Prokopenko, B. V. Shvetsov. The current test procedure for heating appliances - is there an adjustment? ABOK No. 4, 2007.

9. G.A. Bershidsky, V.I. Sasin, V.A. Sotchenko. Method for determining the nominal heat flux of heating devices with a water coolant. - M.: NIIsanotechnika, 1984.

10. Sargsyan S.V. Investigation of the ways to organize the air exchange and air difuusion systems on physical models in the laboratory conditions // Scientific review. - 2015. No. $16 .-$ p. $68-71$.

11. Sargsyan S.V. Methods of laboratory testing of the procedures of the air exchange organization on physical models // Scientific review. - 2015. - No. 16. - p. 76-79.

12. S.V. Sargsyan, V.A. Borisova. Mathematical model of spatial heat exchange of rooms with linearly sources of heat. Vladivostok. 2018 